\title{
Fibrotic response to angiotensin II is blunted in the kidney, but not in the heart, in insulin-sensitive long-lived transgenic dwarf rats
}

\author{
OSAMI SATO ${ }^{1}$, NAOTO ASHIZAWA ${ }^{1}$, AKIRA OHTSURU ${ }^{2}$, RYO IMANISHI ${ }^{1}$, HIROAKI KAWANO ${ }^{1}$, \\ SHINJI SETO ${ }^{1}$, SHUNICHI YAMASHITA ${ }^{2}$, ISAO SHIMOKAWA ${ }^{3}$ and KATSUSUKE YANO ${ }^{1}$ \\ ${ }^{1}$ Division of Cardiovascular Medicine, Course of Medical and Dental Sciences, ${ }^{2}$ Division of Molecular \\ Medicine, Course of Life Sciences and Radiation Research, ${ }^{3}$ Department of Pathology and \\ Gerontology, Graduate School of Biomedical Sciences, Nagasaki University, Nagasaki, Japan
}

Received August 28, 2006; Accepted October 13, 2006

\begin{abstract}
Insulin resistance is a characteristic feature of cardiovascular and renal diseases, and angiotensin II (Ang II) has been suggested to induce insulin resistance. The aims of this study were to elucidate the effect of chronic Ang II infusion on vascular reactivity and organ damage in insulinsensitive rats. We confirmed the following three points. First, there was no significant difference in pressor response to chronic Ang II infusion (600 $\mathrm{ng} / \mathrm{kg} / \mathrm{min}$ ) between insulinsensitive transgenic rats (Tg) and control rats (C). Second, there was no significant difference in cardiac hypertrophy and fibrosis by chronic Ang II infusion between the two groups. However, third, fibrotic response to chronic Ang II infusion evaluated by histopathological scoring in the kidney was significantly decreased in insulin-sensitive transgenic rats (renal fibrosis and nephropathy score: C+Ang II vs Tg+Ang II; 2.5 vs 1.3 ; $\mathrm{p}<0.05$ ). Furthermore, the expression of TGF- $\beta$, a fibrosis indicator, was also significantly suppressed in the kidneys of the transgenic rats (TGF- $B 1$ / GAPDH ratio: $\mathrm{C}+$ Ang II vs $\mathrm{Tg}+\mathrm{Ang}$ II; 1.15 vs $0.81 ; \mathrm{p}<0.05$ ). This result indicates that the growth hormone/insulin-like growth factor-1 axis is critically involved in the development of renal injury and fibrosis, rather than hypertension, cardiac hypertrophy, and cardiac fibrosis induced by chronic Ang II administration.
\end{abstract}

Correspondence to: Dr Naoto Ashizawa, Division of Cardiovascular Medicine, Course of Medical and Dental Sciences, Graduate School of Biomedical Sciences, Nagasaki University, 1-7-1 Sakamoto, Nagasaki City, Nagasaki 852-8501, Japan

E-mail: hirorin@net.nagasaki-u.ac.jp

Key words: angiotensin II, insulin sensitivity, growth hormone, fibrosis, kidney

\section{Introduction}

We have previously reported a long-lived transgenic dwarf rat model in which the growth hormone $(\mathrm{GH})$ /insulin-like growth factor (IGF)-1 axis was selectively suppressed by the overexpression of the antisense GH transgene (tg). This transgenic rat is unique in having a longer lifespan, mainly due to a reduced prevalence of cardiovascular and nephropathic events, smaller body size, and an increased insulin sensitivity $(1,2)$. Recently, GH antagonist transgenic mice demonstrated a marked reduction in circulating IGF-1, blood glucose, and insulin levels, and an increased peripheral insulin sensitivity as measured by an insulin tolerance test and hyperinsulinemic euglycemic clamp analysis, suggesting that the chronic elevation of GH levels plays an important role in insulin resistance (3). Diminished insulin sensitivity is a characteristic feature of various pathological conditions such as hypertension, heart failure, and type 2 diabetes $(4,5)$. In these pathological conditions, angiotensin II (Ang II) has been suggested to induce insulin resistance. Moreover, increased target organ damage in the cardiovascular system and pressor responsiveness to Ang II were found in insulinresistant hypertensive rats. In the heart, Ang II induces cardiac hypertrophy, perivascular and interstitial fibrosis, and oxidative stress, which have been linked to end-stage heart failure $(6,7)$. Furthermore, renal cell growth and extracellular matrix (ECM) synthesis and degradation were modulated by Ang II, leading to glomerulosclerosis and interstitial fibrosis and consequently to advanced renal failure (8). It has also been shown that ACE inhibitor and the Ang II receptor blocker improve insulin sensitivity and reduce blood pressure in essential hypertension and fructose-induced hyperinsulinemic hypertensive rats (9-11). However, it is not clear whether a blunted response to Ang II is found in insulinsensitive animal models. Accordingly, our insulin-sensitive rats may have diminished responses to Ang II, which can be linked to their reduced prevalence of cardiovascular and nephropathic complications, and subsequently to their longevity. In the present study, we examined the effect of chronic Ang II infusion on vascular reactivity and organ 
damage in long-lived transgenic dwarf rats compared with control rats.

\section{Materials and methods}

Animal model. The transgenic rats [Jcl:Wistar-TgN (ARGHGEN)1Nts] were kindly provided by Nippon Institute for Biological Science (Oume City, Tokyo, Japan) and the present colony has been established in a barrier facility in the Laboratory Animal Center at Nagasaki University School of Medicine since 1997. The transgene consisted of four copies of the thyroid hormone response element, the rat GH promoter, and antisense cDNA sequences for rat GH. Sixteen-week-old male heterozygous (tg/-) rats and male Wistar control (-/-) rats (Japan Clea Inc., Tokyo, Japan) were used in this study. Because homozygous ( $\mathrm{tg} / \mathrm{tg}$ ) rats do not have long lives due to the increased prevalence of leukemia and other neoplastic disease, we used heterozygous (tg/-) rats as a model of longlived insulin-sensitive rats. The following study was approved by the Animal Rights Committee, Nagasaki University.

Ang II infusion. Ang II (600 ng/kg/min) was continuously administered to heterozygous (tg/-) rats $(\mathrm{n}=8)$ and control (-/-) rats $(n=8)$ for 4 weeks via a subcutaneous-implanted osmotic mini-pump (Alzet model 2002, Palo Alto, CA). As a vehicle control, saline was continuously administered to heterozygous $(\mathrm{tg} /-)$ rats $(\mathrm{n}=8)$ and control (-/-) rats $(\mathrm{n}=8)$ for 4 weeks. Ang II (Sigma Aldrich Chemicals Japan, Tokyo, Japan) was dissolved in saline at $4 \mathrm{mg} / \mathrm{ml}$, and acetic acid was added to maintain its stability (final concentration, $0.01 \mathrm{~mol} / \mathrm{l}$ ).

Blood pressure, heart rate, and body weight measurements. Blood pressure (BP) and heart rate (HR) were measured in conscious rats by the tail-cuff method (Model MK-2000, Muromachi Kikai Co, Tokyo, Japan) and body weight (BW) was recorded before and after 4 weeks of Ang II or saline infusion.

Echocardiography. At 4 weeks of treatment, echocardiography was performed under pentobarbital anesthesia (50 mg $/ \mathrm{kg}$, ip). A Toshiba Aplio echocardiograph machine (SSA$700 \mathrm{~A}$ ) equipped with a $7.5-\mathrm{MHz}$ phased-array transducer
RWT
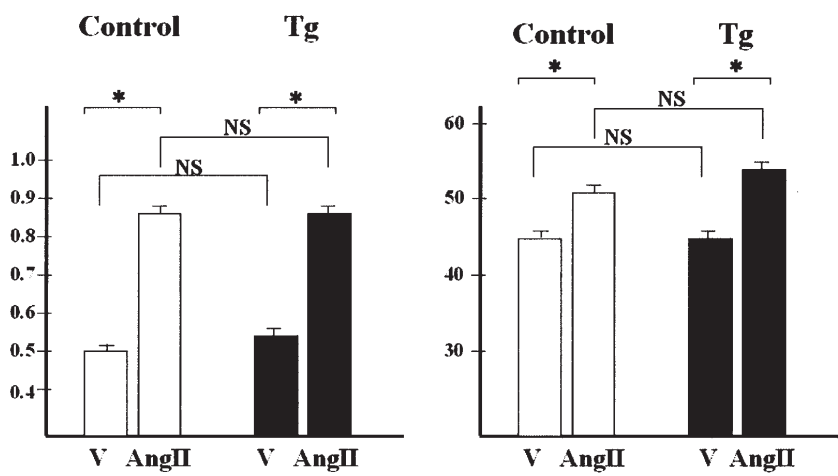

Figure 1. Transthoracic echocardiographic findings in the control and transgenic rats. Open bars, control (-/-) rats; solid bars, transgenic (tg/-) rats; ${ }^{*} \mathrm{p}<0.05$ compared with those in the respective vehicle-infused groups. Ang II, angiotensin II; V, vehicle; FS (\%), fractional shortening; and RWT, relative wall thickness.

was used. Anterior and posterior wall thickness (PWT) and left ventricle (LV) internal dimensions were measured. Systolic function was assessed by calculating endocardial fractional shortening (FS). Relative wall thickness (RWT) was calculated as 2xPWT/LV internal dimension $(12,13)$.

Blood sampling and organ collection. At the end of the experiment, the blood of the rats was collected in chilled plastic tubes containing $0.5 \mathrm{ml}$ of $3.8 \%$ EDTA. Plasma IGF-1 level was measured by radioimmunoassay. The heart and right kidney were dissected in each rat. One part of these specimens was fixed in $10 \%$ formalin for histopathological examination and another part was frozen in liquid nitrogen and kept at $-80^{\circ} \mathrm{C}$ until homogenized.

Histological analysis. The paraffin-embedded sections were prepared and stained either with hematoxylin and eosin or Masson's trichrome for light microscopy analysis. Fibrosis in the heart was graded from 0-4 as determined by perivascular

Table I. Animal characteristics and serum IGF-1 after 4 weeks of Ang II infusion.

\begin{tabular}{lcccc}
\hline & $\mathrm{C}+\mathrm{V}(\mathrm{n}=8)$ & $\mathrm{C}+$ Ang II $(\mathrm{n}=8)$ & $\mathrm{Tg}+\mathrm{V}(\mathrm{n}=8)$ & Tg+Ang II (n=8) \\
\hline Systolic blood pressure (mmHg) & $124.4 \pm 2.7$ & $223.4 \pm 12.9^{\mathrm{a}}$ & $133.0 \pm 3.7$ & $218.7 \pm 5.2^{\mathrm{b}}$ \\
Heart rate (bpm) & $468.4 \pm 14.7$ & $462.3 \pm 8.9$ & $411.6 \pm 5.7$ & $454.5 \pm 21.7$ \\
Body weight (g) & $461.1 \pm 7.3$ & $340.0 \pm 7.1^{\mathrm{a}}$ & $336.6 \pm 9.4^{\mathrm{c}}$ & $272.8 \pm 5.4^{\mathrm{b}, \mathrm{d}}$ \\
Heart weight/body weight (mg/g) & $2.40 \pm 0.06$ & $3.31 \pm 0.14^{\mathrm{a}}$ & $2.82 \pm 0.07$ & $3.54 \pm 0.08^{\mathrm{b}}$ \\
Kidney weight/body weight $(\mathrm{mg} / \mathrm{g})$ & $2.84 \pm 0.18$ & $3.55 \pm 0.14^{\mathrm{a}}$ & $2.80 \pm 0.08$ & $3.51 \pm 0.06^{\mathrm{b}}$ \\
Serum IGF-1 (ng/ml) & $790 \pm 26$ & $484 \pm 60^{\mathrm{a}}$ & $487 \pm 19^{\mathrm{c}}$ & $346 \pm 41$ \\
\hline
\end{tabular}

Data are expressed as mean \pm SEM. C, control group; V, vehicle-infused group; Ang II, Angiotensin II-infused group; and Tg, transgenic group. ${ }^{\mathrm{a}}<0.05, \mathrm{C}+\mathrm{V}$ groups vs $\mathrm{C}+\mathrm{Ang}$ II groups; ${ }^{\mathrm{b}} \mathrm{P}<0.05, \mathrm{Tg}+\mathrm{V}$ groups vs $\mathrm{Tg}+\mathrm{Ang}$ II groups; ${ }^{\mathrm{c}} \mathrm{P}<0.05, \mathrm{C}+\mathrm{V}$ groups vs $\mathrm{Tg}+\mathrm{V}$ groups; and ${ }^{\mathrm{d}} \mathrm{P}<0.05, \mathrm{C}+$ Ang II groups vs Tg+Ang II groups. 


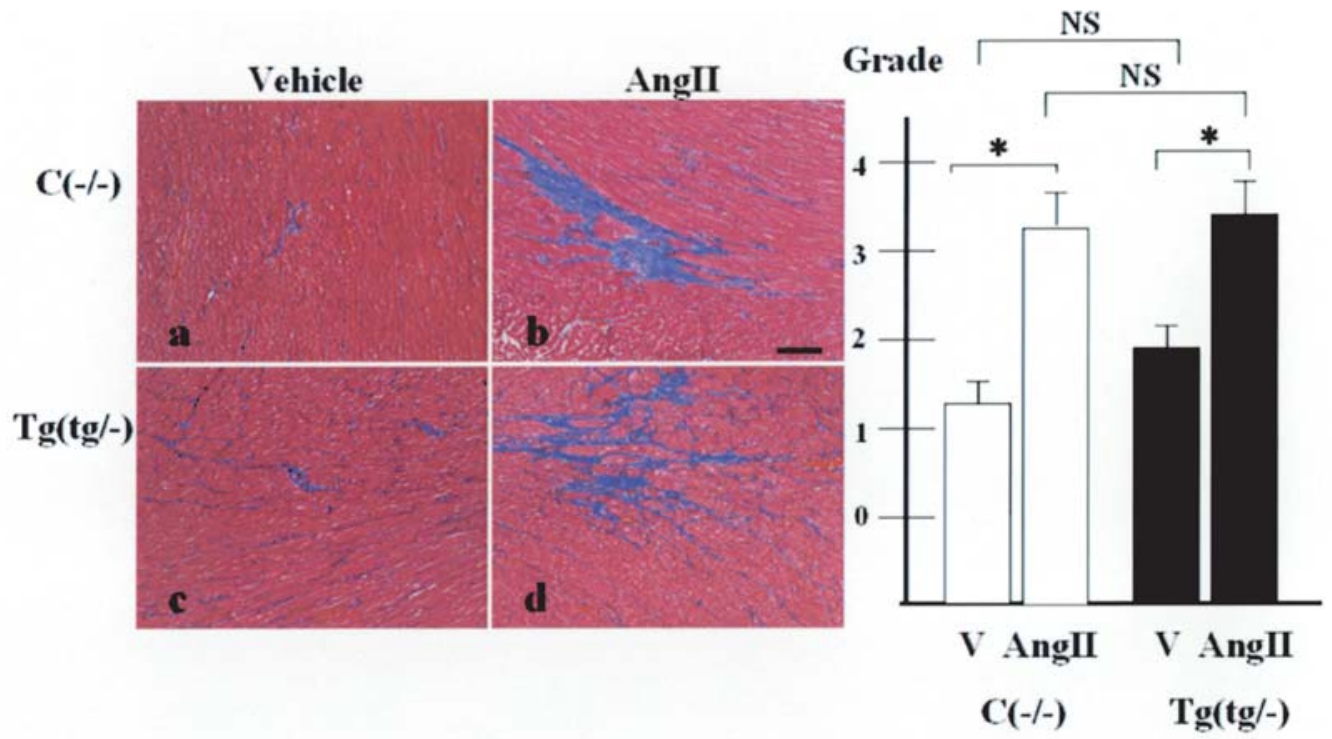

Figure 2. Light microscopy photomicrographs showing representative images of ventricle stained with Masson's trichrome corresponding to the control rat: vehicle infusion (a) and Ang II infusion (b); and the Tg (tg/-) rat: vehicle infusion (c) and Ang II infusion (d). Bar is $100 \mu \mathrm{m}$. Grading of cardiac fibrosis was defined as follows: grade 0, no lesion; grade 1, perivascular fibrosis; grade 2, perivascular fibrosis + myocardial fibrosis, minimal; grade 3, perivascular fibrosis + myocardial fibrosis, mild; and grade 4 , perivascular fibrosis + myocardial fibrosis, moderate. * $\mathrm{p}<0.05$ compared with those in the respective vehicle-infused groups.

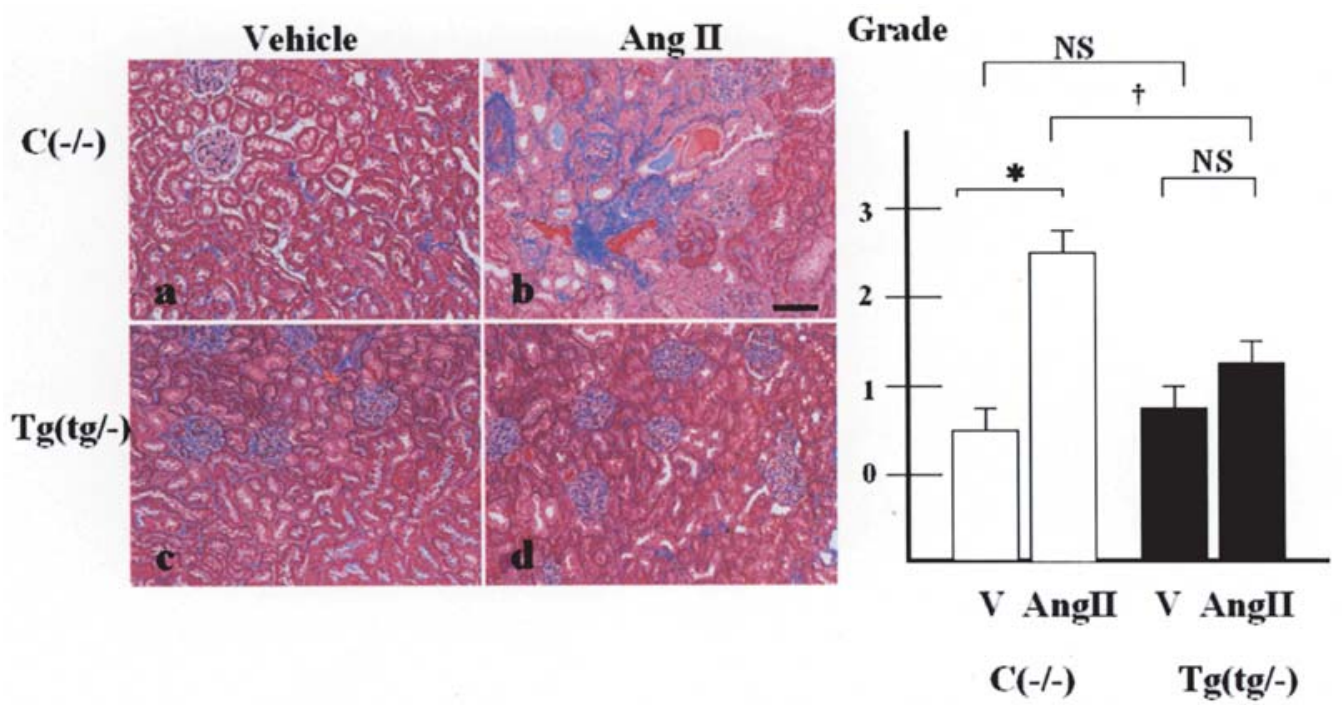

Figure 3. Light microscopy photomicrographs showing representative images of kidney stained with Masson's trichrome corresponding to control rat: vehicle infusion (a) and Ang II infusion (b); and the Tg (tg/-) rat: vehicle infusion (c) and Ang II infusion (d). Bar is $100 \mu \mathrm{m}$. Grading of renal involvement was defined as follows: grade 0 , no lesion; grade 1, interstitial fibrosis, mild; grade 2, interstitial fibrosis, mild + nephropathy, mild and grade 3 , interstitial fibrosis, moderate + nephropathy, moderate. ${ }^{*} \mathrm{p}<0.05$ compared with those in the respective vehicle-infused groups. ${ }^{\dagger} \mathrm{p}<0.05$ compared with those in the respective Ang II-infused groups.

fibrosis and myocardial fibrosis. Renal involvement was graded from 0-3 as determined by interstitial fibrosis and nephropathy. The severity of nephropathy was scored using the method of Maeda and colleagues with some modifications $(14,15)$.

Semi-competitive RT-PCR. Total RNA was isolated from the kidney and ventricle using Trizol reagent (Invitrogen Japan, Tokyo, Japan) according to the manufacturer's instructions. Reverse transcription followed by polymerase chain reaction (RT-PCR) was performed using a Qiagen OneStep RT-PCR kit (Qiagen Japan, Tokyo, Japan) according to the manufacturer's protocol. Primers used were as follows: TGF- $\beta 1$, 5'-AAG AAC TGC TGT GTG CGG-3' and 5'-GCA CTT GCA GGA GCG CAC AA-3' (296 bp); and GAPDH, 5'AGA TCC ACA ACG GAT ACA TT-3' and 5'-TCC CTC AAG ATT GTC AGC AA-3' (309 bp) $(16,17)$. Quantitative analysis was performed by NIH imaging.

Statistical analysis. Data were represented as the mean \pm SEM. One-way analysis of variance (ANOVA) followed by the Scheffe's F-test were performed for statistical comparisons. Differences were considered statistically significant when $\mathrm{p}<0.05$. 


\section{RT-PCR}
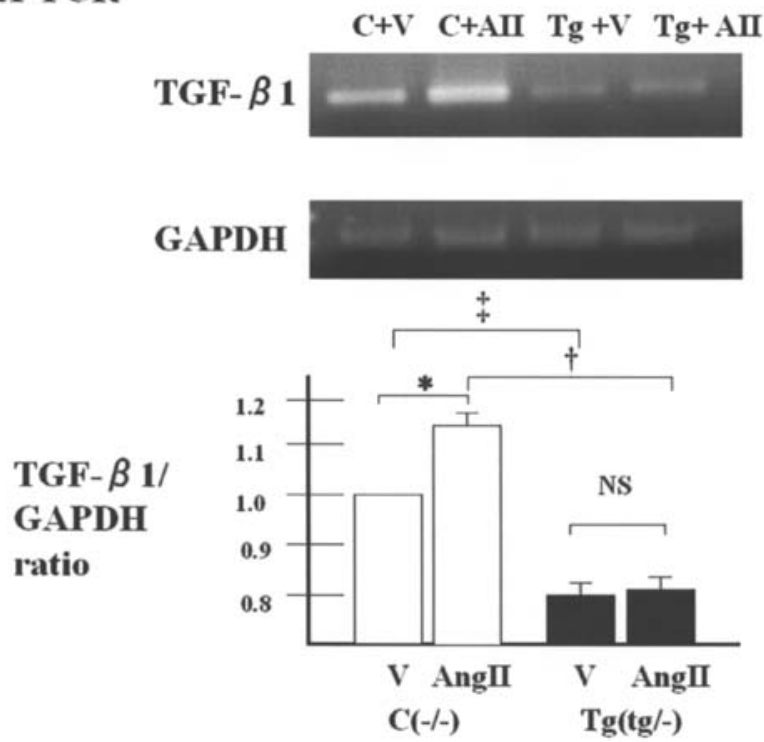

Figure 4. RT-PCR analysis of renal TGF- $\beta 1$ expression in control + vehicle, control + Ang II, Tg (tg/-)+vehicle and Tg (tg/-) + Ang II. ${ }^{*} \mathrm{p}<0.05$ compared with those in the respective vehicle-infused groups. ${ }^{\dagger} \mathrm{p}<0.05$ compared with those in the respective Ang II-infused groups. ${ }^{*} \mathrm{p}<0.05$ compared vehicle-infused control groups with those in the respective transgenic groups.

\section{Results}

Animal characteristics and IGF-1 determination. Baseline $\mathrm{BP}$ and HR readings were similar in heterozygous $(\mathrm{tg} /-)$ $(124.9 \pm 2.7 \mathrm{mmHg}, 436.8 \pm 8.3 \mathrm{bpm})$ and control $(-/-)$ $(118.5 \pm 4.5 \mathrm{mmHg}, 448.3 \pm 10.1 \mathrm{bpm})$ rats. BP, HR, BW, heart weight, kidney weight, and serum levels of IGF-1 after 4 weeks of Ang II or vehicle infusion to transgenic and control rats are shown in Table I. Ang II infusion increased $\mathrm{BP}$ in both the control and transgenic groups to the same extent. Body weight was lighter in the transgenic rats than in the control rats, while rats that received Ang II infusion showed lighter BW than those that received vehicle infusion. To note, transgenic rats had a higher heart weight/BW ratio than control rats during vehicle infusion (statistically insignificant, $\mathrm{p}=0.06$ ), although Ang II infusion increased the heart weight/BW ratio to the same extent in transgenic and control rats. The kidney weight/BW ratio also increased by Ang II infusion in both transgenic and control rats. Similar kidney weight/BW ratios were observed between transgenic and control rats either with Ang II or vehicle infusion. Ang II reduced serum IGF-1 level in both transgenic and control rats.

Echocardiography. As shown in Fig. 1, RWT and \%FS in the Ang II-infused transgenic and control rats (C+Ang II and $\mathrm{Tg}+$ Ang II) were significantly higher than those in the respective vehicle-infused rats $(\mathrm{C}+\mathrm{V}$ and $\mathrm{Tg}+\mathrm{V})$. Both control and transgenic rats showed a marked increase in RWT and \%FS after Ang II infusion.

Histological analysis. Fig. 2 indicates that there was no difference in cardiac fibrosis between Ang II-infused transgenic and control rats (C+Ang II and $\mathrm{Tg}+\mathrm{Ang}$ II) and their respective vehicle-infused rats $(\mathrm{C}+\mathrm{V}$ and $\mathrm{Tg}+\mathrm{V})$ (cardiac fibrosis score C+Ang II vs $\mathrm{Tg}+\mathrm{Ang}$ II; 3.3 vs 3.5; statistically insignificant). However, as shown in Fig. 3, the severity of renal involvement was remarkably higher in Ang IIinfused control rats (C+Ang II) than in those of Ang IIinfused transgenic rats (Tg+Ang II) (renal fibrosis and nephropathy score: C+Ang II vs $\mathrm{Tg}+\mathrm{Ang}$ II; 2.5 vs 1.3 ; $\mathrm{p}<0.05)$.

Expression of TGF- $\beta 1$ mRNA in the kidney. To examine whether the fibrotic response in the kidney by Ang II infusion was blunted in transgenic rats, RT-PCR analysis of TGF- $\beta 1$, as a marker of fibrosis, was performed. As shown in Fig. 4, the expression of the mRNA level of TGF- $\beta 1$ was significantly higher in control rats (C+Ang II) compared to transgenic rats (Tg+Ang II) after Ang II infusion (TGF-ß1/GAPDH ratio: C+Ang II vs Tg+Ang II; 1.15 vs $0.81 ; p<0.05$ ).

\section{Discussion}

In the present study, we first confirmed the effects of the chronic administration of Ang II on pressor response as well as morphological and histological changes in the heart and the kidney in an insulin-sensitive transgenic rat model. Recently, many reports concerning growth hormone (GH)suppressed rodents have documented longevity, small size, and insulin hypersensitivity. Insulin hypersensitivity was also demonstrated using a glucose tolerance test and glucose clamp test $(18,19)$. It is well recognized that the reninangiotensin system (RAS) plays an important physiological role in body fluid and sodium homeostasis and blood pressure regulation, whereas RAS activation causes hypertension and other cardiovascular and renal diseases (20). Experimental and clinical studies have demonstrated the benefit of the RAS blockade in the treatment of such cardiovascular and renal diseases (21-24). Bunnag et al (25) suggested that the blood pressure response to an acute infusion of Ang II was not significantly different among high fructose-fed rats, high sucrose-fed rats, and control rats. However, Gaboury et al (26) confirmed that hypertensive subjects but not normotensive subjects display a striking negative correlation between pressor response to Ang II and insulin resistance from the results of acute Ang II infusion and a glucose clamp test. Although it is not clear whether the response to chronic Ang II infusion is enhanced in insulinresistant animal models, there are studies showing that the administration of the Ang II receptor blockade reduces or abolishes blood pressure elevation and hyperinsulinemia in insulin-resistant rats $(27,28)$. Thus, we assumed that a blunted response to chronic Ang II would be found in our insulinsensitive transgenic rats. In the present study, we confirmed the following three points. First, there was no significant difference in pressor response to chronic Ang II infusion between insulin-sensitive transgenic and control rats. Second, there was no significant difference in cardiac hypertrophy and fibrosis by chronic Ang II infusion between the two groups. However, third, fibrotic response to chronic Ang II infusion in the kidney was significantly decreased in insulinsensitive transgenic rats. Generally, continuous Ang II infusion has been shown to cause blood pressure elevation, 
cardiac hypertrophy, and renal dysfunction in animal models. In this study, Ang II infusion induced marked blood pressure elevation and hypertrophic responses, including increases in heart weight and LV wall thickness in both groups. In contrast, the preventive effect of GH/IGF-1 axis suppression on renal fibrosis and injury induced by Ang II was identified in insulin-sensitive transgenic rats. Furthermore, expression of TGF- $\beta$, a fibrosis indicator, was also significantly suppressed in the kidneys of the transgenic rats. This result indicated that the GH/IGF-1 axis was critically involved in the development of renal injury and fibrosis, rather than hypertension, cardiac hypertrophy, and cardiac fibrosis induced by Ang II. The mechanism of this difference by chronic Ang II infusion observed in the heart and kidneys is not clear at present. Anderson et al (29) suggested that insulin, itself, significantly increases TGF- $\beta$ and extracellular matrix gene expression in rat mesangial cells. However, Ang II alone has modest effects, while Ang II and insulin have additive effects. They also suggested that enhancement of mitogen-activated protein (MAP) kinase activity and AT1 receptor message level by insulin may contribute to the additive effects of insulin and Ang II in rat mesangial cells. Moreover, Brown-Borg et al (30) suggested that the reduced levels of glutathione peroxidase (GPX) proteins, one family of antioxidant proteins, in the heart and skeletal muscle were modest in comparison to those observed in the liver and kidney in Ame dwarf mice by growth hormone administration. The organ damage difference between the heart and kidney may be explained by the organ-specific response under oxidative stress. Further study is required to elucidate the differences in organ damage between the heart and kidneys with regard to signal transduction below the level of insulin/IGF-1 and Ang II receptors. In conclusion, insulin hypersensitivity with GH/ IGF-1 axis suppression ameliorates renal fibrosis and injury caused by excessive Ang II. Application of these results clinically may lead to a promising new treatment for hypertension, heart failure and type 2 diabetes.

\section{References}

1. Shimokawa I, Higami Y, Tsuchiya T, et al: Life span extension by reduction of the growth hormone-insulin-like growth factor-1 axis: relation to caloric restriction. FASEB J 17: 1108-1109, 2003.

2. Shimokawa I, Fukuyama T, Yanagihara-Outa K, et al: Effects of caloric restriction on gene expression in the arcuate nucleus. Neurobiol Aging 24: 117-123, 2003.

3. Yakar S, Setser J, Zhao H, et al: Inhibition of growth hormone action improves insulin sensitivity in liver IGF-1-deficient mice. J Clin Invest 113: 96-105, 2004.

4. Clark CM Jr and Lee DA: Prevention and treatment of the complications of diabetes mellitus. N Engl J Med 332: 1210-1217, 1995.

5. Reaven GM, Lithell $\mathrm{H}$ and Landsberg L: Hypertension and associated metabolic abnormalities - the role of insulin resistance and the sympathoadrenal system. N Engl J Med 334: 374-381, 1996.

6. Luft FC, Mervaala E, Muller DN, et al: Hypertension-induced end-organ damage: A new transgenic approach to an old problem. Hypertension 33: 212-218, 1999.

7. Kamide K, Rakugi H, Higaki J, et al: The renin-angiotensin and adrenergic nervous system in cardiac hypertrophy in fructosefed rats. Am J Hypertens 15: 66-71, 2002.

8. Mezzano SA, Ruiz-Ortega M and Egido J: Angiotensin II and renal fibrosis. Hypertension 38: 635-638, 2001.
9. Iimura O, Shimamoto K, Matsuda K, et al: Effects of angiotensin receptor antagonist and angiotensin converting enzyme inhibitor on insulin sensitivity in fructose-fed hypertensive rats and essential hypertensives. Am J Hypertens 8: 353-357, 1995.

10. Navarro-Cid J, Maeso R, Perez-Vizcaino F, et al: Effects of losartan on blood pressure, metabolic alterations, and vascular reactivity in the fructose-induced hypertensive rat. Hypertension 26: 1074-1078, 1995.

11. Fang TC and Huang WC: Angiotensin receptor blockade blunts hyperinsulinemia-induced hypertension in rats. Hypertension 32: 235-242, 1998.

12. Cittadini A, Stromer H, Katz SE, et al: Differential cardiac effects of growth hormone and insulin-like growth factor-1 in the rat. A combined in vivo and in vitro evaluation. Circulation 93: 800-809, 1996.

13. Imanishi R, Ashizawa N, Ohtsuru A, et al: GH suppresses TGFbeta-mediated fibrosis and retains cardiac diastolic function. Mol Cell Endocrinol 218: 137-146, 2004.

14. Maeda H, Gleiser CA, Masoro EJ, Murata I, McMahan CA and Yu BP: Nutritional influences on aging of Fischer 344 rats: II. Pathology. J Gerontol 40: 671-688, 1985.

15. Shimokawa I, Higami Y, Utsuyama M, et al: Life span extension by reduction in growth hormone-insulin-like growth factor-1 axis in a transgenic rat model. Am J Pathol 160: 2259-2265, 2002.

16. Chen WS and Sim MK: Effects of des-aspartate-angiotensin I on the expression of angiotensin AT1 and AT2 receptors in ventricles of hypertrophic rat hearts. Regul Pept 117: 207-212, 2004.

17. Tohma T, Shimabukuro M, Oshiro Y, Yamakawa M, Shimajiri Y and Takasu N: Cilostazol, a phosphodiesterase inhibitor, reduces microalbuminuria in the insulin-resistant Otsuka LongEvans Tokushima Fatty rat. Metabolism 53: 1405-1410, 2004.

18. Bartke A and Brown-Borg H: Life extension in the dwarf mouse. Curr Top Dev Biol 63: 189-225, 2004.

19. Bartke A: Minireview: role of the growth hormone/insulin-like growth factor system in mammalian aging. Endocrinology 146: 3718-3723, 2005.

20. Morgan T: Renin, angiotensin, sodium and organ damage. Hypertens Res 26: 349-354, 2003.

21. Kobayashi S, Moriya H, Nakabayashi I, Nishiyama J and Fukuda T: Angiotensin II and IGF-I may interact to regulate tubulointerstitial cell kinetics and phenotypic changes in hypertensive rats. Hypertens Res 25: 257-269, 2002.

22. Okada H, Watanabe $\mathrm{Y}$, Inoue $\mathrm{T}$, et al: Angiotensin II type 1 receptor blockade attenuates renal fibrogenesis in an immunemediated nephritic kidney through counter-activation of angiotensin II type 2 receptor. Biochem Biophys Res Commun 314: 403-408, 2004.

23. Xu ZG, Lanting L, Vaziri ND, et al: Upregulation of angiotensin II type 1 receptor, inflammatory mediators, and enzymes of arachidonate metabolism in obese Zucker rat kidney: reversal by angiotensin II type 1 receptor blockade. Circulation 111: 1962-1969, 2005.

24. Yamazaki T, Komuro I and Yazaki Y: Role of the reninangiotensin system in cardiac hypertrophy. Am J Cardiol 83: $53 \mathrm{H}-57 \mathrm{H}, 1999$.

25. Bunnag P, Hori MT, Ormsby B, Berger ME, Golub MS and Tuck ML: Impaired in vivo adrenergic responses in diet-induced hypertensive rats. Hypertens Res 20: 17-21, 1997.

26. Gaboury CL, Simonson DC, Seely EW, Hollenberg NK and Williams GH: Relation of pressor responsiveness to angiotensin II and insulin resistance in hypertension. J Clin Invest 94: 2295-2300, 1994.

27. Higashiura K, Ura N, Takada T, et al: The effects of an angiotensin-converting enzyme inhibitor and an angiotensin II receptor antagonist on insulin resistance in fructose-fed rats. Am J Hypertens 13: 290-297, 2000.

28. Oda T, Hirata M, Oshida Y, Han YQ, Koshinaka K and Sato Y: Effect of imidapril, an angiotensin-converting enzyme inhibitor, on fructose-induced insulin resistance in rats. Endocr J 51: 69-74, 2004.

29. Anderson PW, Zhang XY, Tian J, et al: Insulin and angiotensin II are additive in stimulating TGF-beta 1 and matrix mRNAs in mesangial cells. Kidney Int 50: 745-753, 1996.

30. Brown-Borg HM and Rakoczy SG: Growth hormone administration to long-living dwarf mice alters multiple components of the antioxidative defense system. Mech Ageing Dev 124: 1013-1024, 2003. 